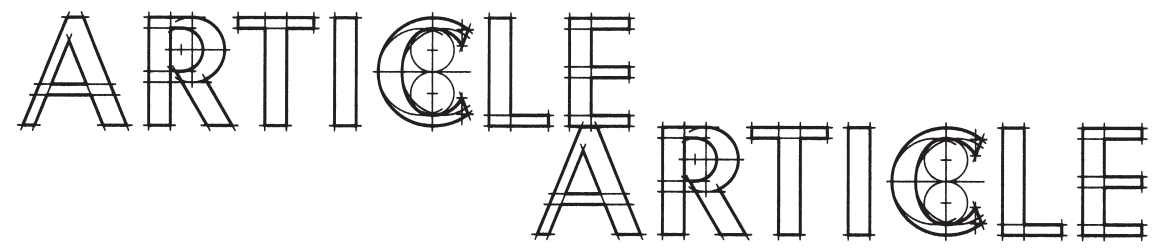

\title{
Caractéristiques et traitements du kératocône
}

\section{Characteristics and treatment of keratoconus}

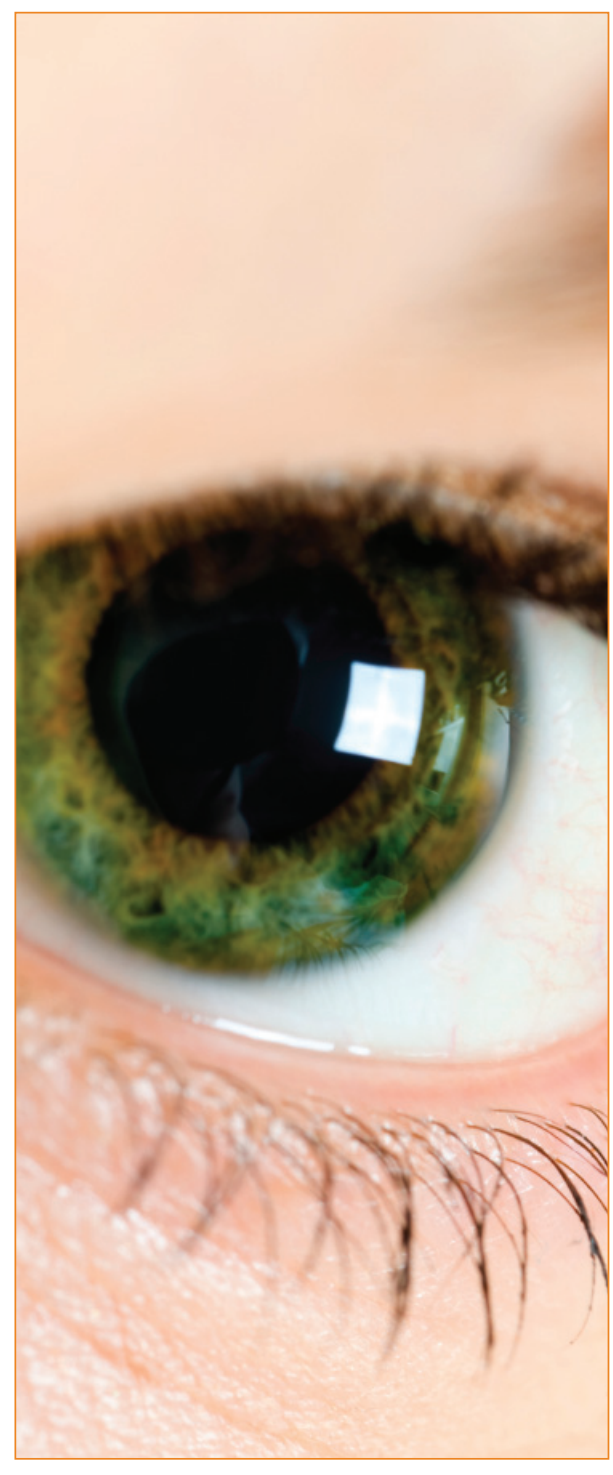

Sommaire: La présente revue de littérature traite $d u$ kératocône, un amincissement progressif de la cornée. Le kératocône induit habituellement de l'astigmatisme irrégulier de sorte que les patients les plus affectés ont une acuité visuelle diminuée limitant ainsi la correction à l'aide de lentilles ophtalmiques. La CLEK (Collaborative Longitudinal Evaluation of Keratoconus) est une étude effectuée dans plusieurs centres dans le but de décrire l'évolution et les associations entre les manifestations visuelles et physiologiques des kératocônes. Étant donné que le traitement du kératocône varie selon la sévérité, le présent article propose un sommaire des résultats du CLEK et un survol des options thérapeutiques.

Abstract: The focus of the present review of literature is keratoconus, a progressive thinning of the cornea. Because keratoconus usually induces irregular astigmatism, the most affected patients experience a decreased visual acuity that limits the correction using ophthalmic lenses. The CLEK (Collaborative Longitudinal Evaluation of Keratoconus) is a study carried out in several centers with the aim of describing the evolution and associations between the visual and physiological manifestations of keratoconus. Since the treatment of keratoconus varies according to its severity, this paper proposes a summary of the results of the CLEK and a review of the therapeutic options.

\section{INTRODUCTION}

Le kératocône est la plus fréquente des dystrophies induisant une ectasie de la cornée. Selon le
Patrick Simard

OD, MSC

École d'optométrie,

Université de Montréal, QC and

Clinique d'Optométrie

Bélanger, Montreal, QC

Claude J. Giasson

$\mathrm{OD}, \mathrm{PhD}, \mathrm{FAAO}$

École d'optométrie,

Université de Montréal, QC and

Laboratoire d'Organogénèse

Expérimentale (LOEX),

Saint-Sacrement Hospital,

Québec, QC

Mots clés: Kératocône, CLEK, Lentilles Cornéennes, Kératoplastie Pénétrante, Anneaux intra-cornéens, LASIK, KPR, Riboflavine

Key words: Keratoconus, CLEK, Contact Lenses, Penetrating Keratoplasty, Intra-corneal Ring, LASIK, PRK, Riboflavin 
« National Keratoconus Institute», l'incidence de cette maladie serait de un sur 2000. Le kératocône est caractérisé par un amincissement progressif du stroma cornéen et une ectasie de la cornée s'écoulant sur plusieurs décennies. Cette maladie débute généralement dans une période très productive de la vie des patients, la vingtaine. Ce processus non-inflammatoire habituellement bilatéral induit de la myopie et de l'astigmatisme irrégulier limitant ainsi la correction avec des lentilles ophtalmiques. Le traitement du kératocône varie en fonction de la sévérité du cas. L'étude CLEK (Collaborative Longitudinal Evaluation of Keratoconus) a été effectuée dans plusieurs centres sur une période de 8 ans dans le but de décrire l'évolution et les associations entre les manifestations visuelles et physiologiques des kératocônes. Plus de mille patients ont été recrutés dans 15 cliniques différentes aux États-Unis. Le présent article représente une partie de la revue de littérature effectuée dans le cadre d'une maitrise sur les densités de kératocytes chez des porteurs de lentilles cornéennes avec ou sans kératocône. Un sommaire des résultats du CLEK suivi d'un survol des options thérapeutiques qui s'offrent aux patients présentant un kératocône insatisfaits de leur condition seront présentés.

\section{Étude multicentrique d'évaluation longitudinale du kératocône (CLEK, Collaborative Longitudinal Evaluation of Keratoconus)}

Les buts généraux du CLEK consistaient à caractériser les changements visuels et cornéens ainsi que la qualité de vie des patients atteints de kératocône. De plus, l'étude voulait décrire la progression des changements dans le temps. A cette fin, les patients étaient d'abord examinés lors de leur recrutement (1995-2006), puis à chaque année par la suite. Lors de chaque visite de chaque patient, les cliniciens du CLEK évaluent les aspects suivants : la qualité de vie reliée à la vision, l'histoire de cas, le temps de port de lentilles cornéennes, l'acuité visuelle, les signes cornéens, les cicatrices cornéennes, la topographie, le mode de traitement, les paramètres de leur ajustement en lentilles perméables aux gaz permettant un dégagement apical. Enfin, la cornée et la relation lentille-cornée par la fluorescéine sont photographiées. Cette accumulation de données sur l'évolution des cas a fait l'objet de plusieurs publications différentes qui sont résumées dans la section suivante.

\section{Les facteurs reliés à la vision}

Le questionnaire sur la fonction visuelle du National Eye Institute (Visual Function Questionnaire, NEI-VFQ) a été rempli à l'examen annuel de suivi par 1166 patients. Ce questionnaire contient 51 échelles différentes. Une acuité visuelle binoculaire inférieure à $6 / 12$ est associée à une faible qualité de vie sur toutes les échelles sauf sur celles de la santé générale et de la douleur oculaire. Des mesures kératométriques supérieures à 52 dioptries (D.) sont corrélées avec de mauvais résultats sur les échelles de la santé mentale (perte de contrôle et inquiétude causée par la vision), de travail (difficulté dans les tâches de vision de près au travail), de conduite automobile (difficulté à conduire le jour et le soir), de dépendance (besoin des autres et nécessité de rester à la maison) et de douleur oculaire (douleur et inconfort autour des yeux). ${ }^{1,2}$ Selon une autre étude, il n'existe aucune association entre la diminution subjective du confort lors du port de lentilles perméables aux gaz et l'augmentation de la sévérité du kératocône mesurée par la courbure kératométrique la plus élevée ou par la première lentille cornéenne permettant un dégagement apical. ${ }^{3}$

Dans une étude multicentrique de cette envergure, il importait de s'assurer de la répétabilité des observations et de la cohérence entre les différents centres. De plus, dans un échantillon portant fréquemment des lentilles cornéennes comme les patients présentant un kératocône, la faisabilité d'évaluer l'ajustement des lentilles cornéennes en fonction du dégagement apical à l'aide d'une photographie et d'une observation directe par le clinicien s'est avérée possible et utile. ${ }^{4,5} \mathrm{La}$ relation lentille-cornée à l'apex cornéen des lentilles cornéennes perméables aux gaz observée en présence de fluorescéine a été évaluée et classifiée en quatre divisions qualitatives: toucher important, toucher, dégagement et dégagement important. La répétabilité et la validité entre les évaluations de ce type faites par les cliniciens lors des visites et par les cliniciens à l'aide de photographies sont excellentes. ${ }^{6}$ La répétabilité des mesures d'acuité visuelle des kératocônes est comparable à celles des normaux. ${ }^{7}$ D'autre part, la répétabilité des réfractions des sujets avec kératocône est inférieure à celle des normaux. Une 


\section{ARTICLE ARTICLE}

surréfraction avec des lentilles cornéennes en place en augmente la répétabilité, mais elle demeure inférieure à une surréfraction effectuée chez des normaux. ${ }^{8}$ Seulement 36\% des réfractions subjectives des sujets avec kératocône donne des mesures sphériques répétables à $+/$ - 0,50 dioptries (D.) comparativement à $90 \%$ pour les sujets normaux. ${ }^{9} \mathrm{Il}$ est important de tenir compte de cette variation clinique de la réfraction lors du choix de la correction à apporter aux patients kératocôniques.

D'autre part, la répétabilité des trois topographes utilisés en mode axial et tangentiel dans cette étude, le EyeSys Model II, le Dicon CT 200 et le Keratron Corneal Analyser, est également diminuée en présence d'une cornée irrégulière kératocônique. ${ }^{10}$ La répétabilité de topographies de cornées avec kératocône à l'aide du topographe Tomey 1 (TMS-1) est également plus faible que celle de normaux. ${ }^{11}$

\section{Les caractéristiques générales des sujets de I'étude CLEK}

L'observation longitudinale de 1209 patients a permis de décrire la fréquence des caractéristiques de ces patients. L'âge moyen au recrutement est de 39,3 +/- 10,9 ans avec un kératocône modéré à sévère. Quatre-vingtquinze pour cent des patients présentent une kératométrie d'au moins 45 dioptries (D.). Soixante-cinq pour cent sont des porteurs bilatéraux de lentilles perméables aux gaz et $73 \%$ de tous les porteurs les trouvent confortables malgré un toucher apical dans $88 \%$ des ajustements. La meilleure acuité visuelle corrigée dans chacun des yeux est de $6 / 12$ ou mieux dans $78 \%$ des cas. Cinquante-trois pour cent ont des cicatrices cornéennes dans au moins l'un des deux yeux. L'opacification cornéenne est associée aux piquetés cornéens, au nombre d'années de port, à la présence d'un anneau de Fleischer et à des cornées plus cambrées. ${ }^{12}$ À l'histoire de cas, $13,5 \%$ ont rapporté la présence de kératocône dans leur famille et $53 \%$ une histoire d'atopie personnelle. ${ }^{13}$ La seule différence entre les hommes et les femmes suite à l'analyse statistique réside dans la présence des stries de Vogt et de l'acuité visuelle monoculaire et binoculaire à haut contraste. Les femmes présentent moins de stries de Vogt et ont une acuité visuelle plus faible. En général, les femmes ont tendance à rapporter plus facilement les symptômes. ${ }^{14}$ Une diminution de l'acuité visuelle à contraste élevé ou bas est observée en présence de cicatrices cornéennes. ${ }^{12,15}$

\section{Les facteurs associés à la présence de} cicatrices cornéennes

L'évaluation de la présence de cicatrices cornéennes par les cliniciens concorde avec l'évaluation faite par des observateurs externes à l'aide des photographies. La prise de photos permettait de s'assurer que tous les cliniciens de l'étude avaient les mêmes critères d'évaluation. ${ }^{16} \mathrm{Le}$ risque de présenter des cicatrices cornéennes est positivement associé avec les facteurs suivants : quantité de cambrure cornéenne, l'âge, la présence de piquetés cornéens ou d'un anneau de Fleischer, le port de lentilles. ${ }^{17}$ Dans ce dernier cas, les porteurs de lentilles cornéennes ont un risque deux fois plus grand de développer des cicatrices cornéennes. Il est important d'éliminer le toucher apical des lentilles cornéennes afin de réduire le risque de développer des cicatrices. ${ }^{18}$ Les figures 1 et 2 illustrent la topographie et l'aspect biomicroscopique d'une cornée provenant d'un patient avec kératocône présentant des cicatrices.

L'augmentation de la courbure cornéenne est reliée à la présence de stries de Vogt, de l'anneau de Fleischer et de cicatrices cornéennes. Ces signes visibles à la biomicroscopie sont plus fréquents, soit environ $60 \%$ des yeux, dans les cornées avec un kératocône jugé sévère. ${ }^{19}$

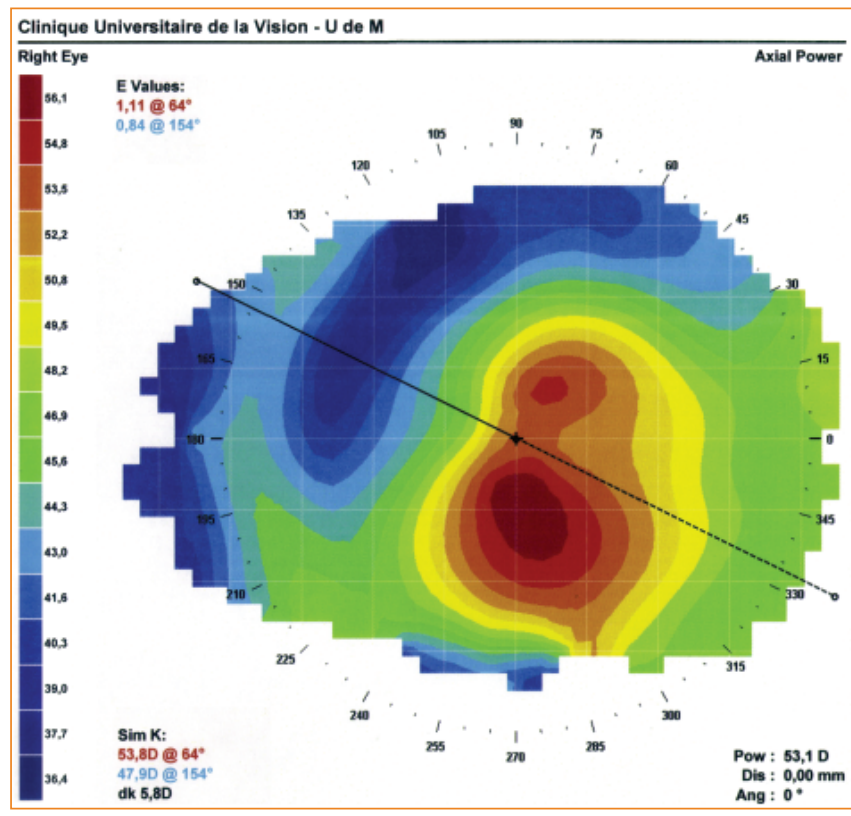

Figure 1. Topographie Medmont d'une cornée cambrée avec kératocône
C A N A D I A N J O U R N A L O F O P T O ME T R Y

R E V U E C A N A D I E N N E D ' O P T O M É T R I E
Vol 70 No 4

July / juillet 2008 


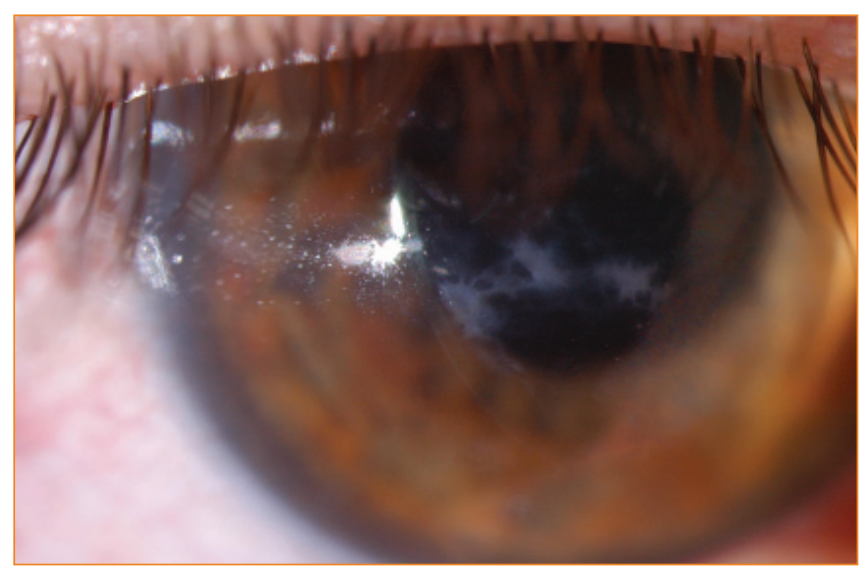

Figure 2. Cicatrices cornéennes chez le même patient de la figure 1

L'asymétrie entre les deux yeux des patients kératocôniques pour la courbure cornéenne, l'acuité visuelle, l'erreur de réfraction et les cicatrices cornéennes est statistiquement significative lorsque comparée à celle des myopes porteurs de lentilles cornéennes. De plus, il existe une association entre le frottement unilatéral des yeux et l'asymétrie; l'œil frotté étant l'œil ayant la courbure la plus élevée. ${ }^{20}$ Les patients avec une forme plus sévère de kératocône sont également plus asymétriques dans la maladie. ${ }^{21}$

Plusieurs études ont observé depuis longtemps la présence dans le stroma cornéen de protéases, enzymes capables de dénaturer les protéines constituant la cornée. L'étude CLEK a adapté au marquage d'un de ces types d'enzymes, les estérases, une technique de transfert des cellules conjonctivales sur une membrane par impression cytologique. Cette technique est préférable au prélèvement d'une biopsie sur toute l'épaisseur conjonctivale. Les spécimens provenant de sujets avec kératocône ont des niveaux d'estérases plus élevés que ceux du groupe témoin, suggérant la présence d'enzymes capables de détruire le tissu cornéen. ${ }^{22}$

D'autre part, contrairement à des publications antérieures indiquant la possibilité que le kératocône soit causé par des anomalies du collagène, les kératocônes ne sont pas associés à un risque accru de souffrir d'une maladie des tissus conjonctifs. ${ }^{13}$ Par ailleurs, l'étude CLEK n'a pas investigué la pathophysiologie du kératocône. Une connaissance accrue des mécanismes induisant le kératocône est nécessaire pour mieux définir les traitements appropriés selon la sévérité des cas.

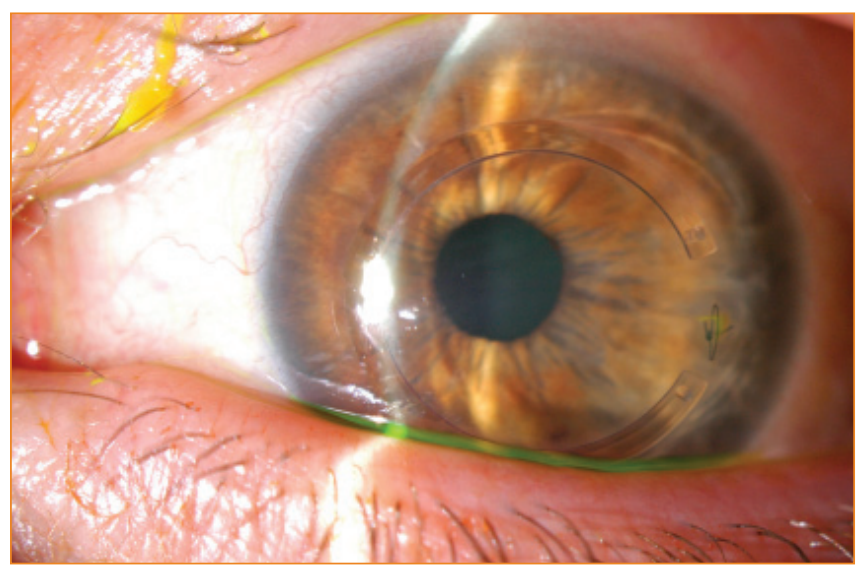

Figure 3. Cornée avec kératocône

\section{Traitement}

La première approche thérapeutique consiste à ajuster des lentilles cornéennes rigides afin de corriger la surface irrégulière de la cornée et d'améliorer la vision, lorsque l'acuité visuelle du patient n'est plus satisfaisante à l'aide de lunettes. Il existe plusieurs types de lentilles perméables aux gaz spécialisées afin d'ajuster des cornées avec kératocône. La description de ces lentilles dépasse le cadre du présent article. Une nouveauté dans le domaine mérite toutefois une mention. Il est maintenant possible de fabriquer des lentilles cornéennes souples sur mesure. Les lentilles prennent en considération les aberrations de l'œil et la topographie cornéenne pour obtenir une correction optimale. ${ }^{23}$

Si le patient devient intolérant aux lentilles perméables aux gaz ou si l'acuité visuelle en déclin ne suffit plus pour remplir les besoins visuels du patient, des lentilles en mode "piggyback » sont parfois prescrites. Ce mode de port couple une lentille molle porteuse, appliquée directement sur la cornée, à une petite lentille perméable aux gaz qui peut se déplacer sur la lentille molle. Cette dernière est parfois usinée de façon à faciliter le centrage de la lentille semi-rigide. L'ensemble permet d'allier donc le confort des lentilles molles à l'acuité visuelle produite par les lentilles perméables aux gaz.

Le plus souvent après l'échec aux lentilles cornéennes, la kératoplastie pénétrante (PK) appelée greffe cornéenne pleine épaisseur est effectuée. Cette procédure consiste à substituer la portion centrale cornéenne d'un patient kératocônique par la cornée saine d'un donneur décédé. La fréquence du kératocône et le temps d'attente

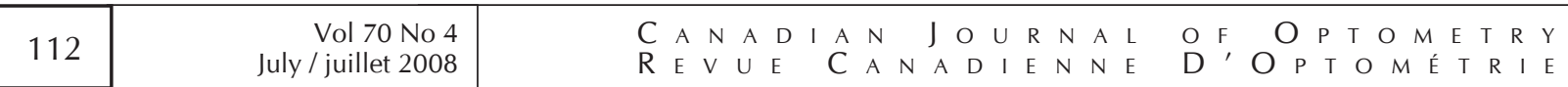




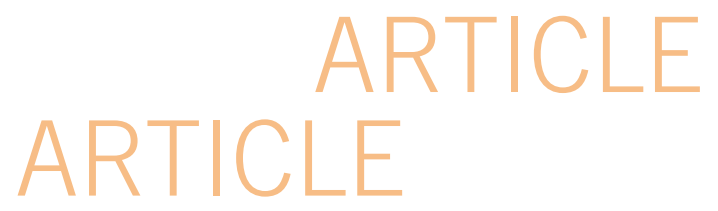

varient selon les pays concernés. Au Canada, une étude rétrospective sur 10 ans montre que le kératocône est la deuxième indication pour la kératoplastie pénétrante avec $10 \%$ des cas après la kératopathie bulleuse du pseudophake. ${ }^{24}$ Le temps d'attente au Québec est d'au moins 2 ans. Au Brésil, le kératocône se classe après les ulcères et la kératopathie bulleuse du pseudophake. ${ }^{25}$ En Chine, le kératocône est la quatrième indication pour une $\mathrm{PK}^{26}$ tout comme aux Etats-Unis. ${ }^{27}$ Le temps moyen écoulé entre le diagnostic du kératocône et la greffe est de 8,5 ans en Angleterre. ${ }^{28}$ Selon une étude effectuée dans un centre de référence tertiaire de Londres, 21,6\% des patients kératocôniques auront besoin d'une greffe cornéenne. ${ }^{29} \mathrm{La}$ meilleure acuité visuelle inférieure à 6/12, un astigmatisme supérieur à $10 \mathrm{D}$., une courbure cornéenne supérieure à $55 \mathrm{D}$., un âge de 30 ans et moins et un diagnostic depuis 5 ans et moins augmentent le risque de nécessiter une greffe cornéenne. ${ }^{30}$ Cependant, le coût important relié à la kératoplastie pénétrante et les aberrations causées par l'astigmatisme irrégulier résultant poussent les chirurgiens à considérer cette approche en dernier recours. D'autres techniques sont maintenant développées afin d'éviter ou de différer la greffe pénétrante.

L’une de ces techniques, plus délicate à exécuter, mais moins sujette à entraîner un astigmatisme important, consiste à ne remplacer qu'une partie de la cornée, la kératoplastie lamellaire. La kératoplastie lamellaire profonde assistée par microkératome consiste à créer une lamelle cornéenne dans la cornée de l'hôte et y introduire le stroma d'un donneur pour ensuite replacer la lamelle. Cette technique permet de conserver l'endothélium de l'hôte. Certains suggèrent d'injecter une bulle d'air dans la chambre antérieure afin de minimiser les risques de perforation avant la formation de la lamelle. ${ }^{31}$ Dans certaines études, une chirurgie réfractive est effectuée 6 mois après la greffe stromale en utilisant un laser excimer afin de corriger l'amétropie du patient. Le traitement au laser est effectué par la méthode de la kératoplastie photoréfractive (KPR) ou du LASIK (Laser in situ keratomileusis), c'est-à-dire en relevant alors la lamelle afin d'exposer le stroma au laser. Les résultats semblent prometteurs, mais les suivis n'ont été effectués que sur une période de 7 à 22 mois. Dans une étude sur 9 yeux de 7 sujets avec kératocône, l'épaisseur cornéenne est passée de 432,7 à $578 \mu \mathrm{m}$ en moyenne après la chirurgie. L'acuité visuelle corrigée de tous les patients s'est améliorée en moyenne de 5 lignes. ${ }^{32}$ Une autre étude sur 50 yeux de 50 patients a mesuré une meilleure acuité visuelle corrigée (MAVC) de 6/12 chez $88 \%$ des patients un an après cette procédure. ${ }^{33}$ Le KPR ou le LASIK n'a pas été effectué dans cette dernière étude. Il faut noter que l'usage des techniques de chirurgie réfractive avec incisions comme le LASIK est loin de faire l'unanimité chez les praticiens. En effet, ces techniques sont contre-indiquées chez les patients avec kératocône, car elles augmentent l'instabilité cornéenne. D'autres approches prometteuses discutées plus loin sont présentement à l'essai.

Une autre approche thérapeutique expérimentale consiste à coloniser un polymère avec des kératocytes pour ensuite espérer remplacer la cornée des patients atteints par le nouveau matériau biocompatible. ${ }^{34}$ Cette dernière technique a été utilisée avec succès chez 4 patients différents dont la cornée était totalement opacifiée. ${ }^{35}$

Une dernière option chirurgicale consiste à introduire des anneaux dans la mi-périphérie du stroma cornéen de patients où la cornée est exempte de cicatrice. Ces anneaux intra-cornéens, en forme de demi-lune permettent de tenter d'aplanir la surface oculaire. Cette technique réversible ${ }^{36}$ 'applique surtout aux kératocônes légers qui ne tolèrent plus les lentilles cornéennes et qui ont une mauvaise acuité visuelle en lunettes. Selon la topographie, la localisation et la grandeur du cône, les chirurgiens implantent un ou deux segments horizontalement en position inférieure ou supérieure. ${ }^{37}$ Les segments doivent être placés asymétriquement par rapport au centre de la cornée pour de meilleurs résultats. ${ }^{38}$ L'implantation dans des cas plus avancés a nécessité l'ajustement d'une lentille cornéenne car la correction apportée par les anneaux intra-cornéens demeurait limitée. ${ }^{39} \mathrm{Il}$ existe deux types d'anneaux commercialisés : Intacs et Ferrara. Les anneaux intra-cornéens peuvent corriger entre $-1,00 \mathrm{D}$ et $-3,00 \mathrm{D}$ et jusqu'à $1,00 \mathrm{D}$ d'astigmatisme. Les résultats de plusieurs des études qui les ont utilisés ont récemment été publiés. ${ }^{38,40-45}$ Cette procédure peut également être effectuée chez des patients souffrant de dégénérescence pellucide marginale. ${ }^{46}$ Sur une période de 12 mois, l'équivalent sphérique de 36 yeux est passé de $-7,29 \mathrm{D}$ à $-4,80 \mathrm{D} .{ }^{47}$ Suite à
C A N A D I A N J O U R N A L O F O P T O METR

R E V U E C A N A D I E N N E D ' O P T O M É T R I E
Vol 70 No 4

July / juillet 2008 
l'implantation des segments chez des patients avec kératocône en attente d'une greffe cornéenne, la meilleure acuité visuelle corrigée est passée en moyenne de 20/50 à 20/32. ${ }^{37}$ Cette procédure limitée permet uniquement d'atténuer les problèmes visuels des patients kératocôniques. La figure 3 présente une cornée avec des anneaux intra-cornéens.

Certains chirurgiens effectuent des kératectomies photo-réfractives (KPR) ou des photokératectomies thérapeutiques (PTK) sur des kératocônes. Même si le kératocône est une contre-indication à la chirurgie réfractive incisive, une équipe de Russie a tenté de corriger la myopie et l'astigmatisme de sujets avec kératocône au moyen d'un traitement par laser excimer. ${ }^{48}$ Comme le kératocône est une anomalie commençant dans le stroma antérieur et progressant graduellement vers le stroma postérieur, l'équipe prétend pouvoir interrompre la progression du cône des cas de kératocônes légers avec le KPR. Ce traitement très controversé permettrait de freiner la progression dans près de $91 \%$ des cas sur une période de suivi de 3,5 ans. ${ }^{48}$, 49 Une autre équipe de Californie effectue la même procédure sur des kératocônes avec des résultats similaires. ${ }^{50,51}$ Un suivi à long terme de ces patients permettra de mieux évaluer la pertinence et l'efficacité réelle de ce traitement, car le risque que les kératocônes traités progressent davantage est présent. En attendant, le PTK peut être utilisé pour enlever une opacité apicale à l'origine d'une intolérance aux lentilles cornéennes sans corriger l'erreur de réfraction. ${ }^{52}$

Une nouvelle approche consiste à introduire une lentille intra-oculaire afin de corriger la myopie chez des sujets avec un kératocône stable. L'astigmatisme résiduel est corrigé en faisant une incision relaxante parallèle au limbe $e^{53}$ ou une kératoplastie avec des anneaux intracornéens. ${ }^{54}$ Des lentilles intra-oculaires toriques peuvent également être introduites dans la chambre antérieure. ${ }^{55}$ Aucun résultat à grande échelle n'est disponible actuellement pour évaluer cette technique.

Dans le but de ralentir la progression des kératocônes, il est possible d'altérer la résistance aux déformations de la cornée. La rigidité de cornées peut être augmentée en modifiant l'entrelacement des fibres de collagène. La méthode utilisée consiste à débrider l'épithélium au centre de la cornée pour appliquer des gouttes de riboflavine (vitamine B2). La cornée est ensuite exposée à un rayonnement d'ultraviolets A (UVA) durant une période de 30 minutes. Cette procédure a préalablement été testée chez des lapins afin d'en vérifier l'innocuité. ${ }^{56}$ Les UVA sont toxiques pour l'endothélium de cornées plus minces que $400 \mu \mathrm{m} .{ }^{57}$ La riboflavine seule n'est pas toxique pour l'endothélium. ${ }^{58}$ Toutefois, une apoptose des kératocytes est observée sur une profondeur antérieure de $50 \mu \mathrm{m} .{ }^{59} \mathrm{La}$ première étude chez l'humain comporte 23 yeux de 22 patients différents. La durée des suivis varie entre 3 mois et 4 ans. Aucun kératocône traité n'a progressé. La kératométrie a diminué de 2,01 $\mathrm{D}$ et l'erreur de réfraction de 1,14 D. dans le cas de 16 yeux. Toutefois, aucune amélioration de l'acuité visuelle n'a été observée. ${ }^{56} \mathrm{D}$ 'autres études sont nécessaires pour placer les balises de cette technique.

Des équipes ont réussi à introduire à l'aide d'un virus ou de plasmides de nouveaux gènes dans les kératocytes de cornées de lapins ou de rats in vivo. ${ }^{60-64}$ D'autres vecteurs ont été utilisés in vitro chez des humains avec succès. ${ }^{65,}{ }^{66}$ Par exemple, l'efficacité de la transduction du gène implanté par des liposomes cationiques dans des kératocytes humains est de 40,5\% ${ }^{66}{ }^{66}$ L'espoir de ce mode de traitement réside dans la possibilité d'identifier les gènes défectueux pour ensuite les modifier.

\section{Conclusion}

La CLEK est une excellente étude descriptive des changements et des caractéristiques des kératocônes. Toutefois, cette étude comporte quelques lacunes. Puisque cette étude fut entamée en 1995, les lentilles cornéennes n'utilisent aucun design contemporain. De plus, l'étude ne traites pas des kératocônes postérieurs, car les topographes utilisés ne pouvaient pas analyser la courbure postérieure de la cornée. Les caractéristiques décrites dans cette étude permettent de mieux évaluer les risques de progression et d'orienter le traitement. La multitude d'options de traitement démontre bien l'hétérogénéité du kératocône. Le kératocône est le résultat final de plusieurs processus différents affectant la cornée de la même façon que plusieurs processus affectent le nerf optique dans le glaucome. Une meilleure compréhension des mécanismes pathophysiologiques est nécessaire pour classer et définir des sous-groupes affectés par cette maladie afin d'en gérer l'évolution et de choisir la meilleure option de traitement. Présentement,

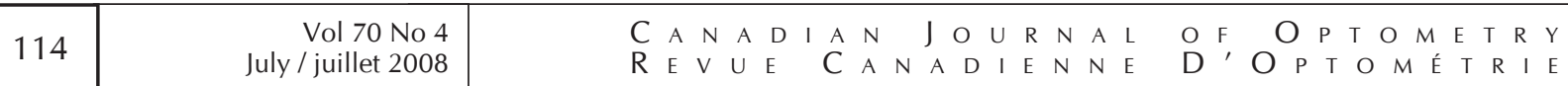


la sélection des traitements s'appuie sur la sévérité des cas sans tenir compte du processus créant la maladie.

\section{Bibliographie}

1. Vitale S. CLEK study reports on the quality of life. Am J Ophthalmol 2004;138:637-638.

2. Kymes SM, Walline JJ, Zadnik K, Gordon MO. Quality of life in keratoconus. Am J Ophthalmol 2004;138:527-535.

3. Edrington TB, Gundel RE, Libassi DP, et al. Variables affecting rigid contact lens comfort in the collaborative longitudinal evaluation of keratoconus (CLEK) study. Optom Vis Sci 2004;81:182-188.

4. Edrington TB, Barr JT, Zadnik K, et al. Standardized rigid contact lens fitting protocol for keratoconus. Optom Vis Sci 1996;73:369-375.

5. Gundel RE, Libassi DP, Zadnik K, et al. Feasibility of fitting contact lenses with apical clearance in keratoconus. Optom Vis Sci 1996;73:729732.

6. Fink BA, Barr JT, Edrington TB, et al. A comparison of two methods of evaluating cornea-to-contact lens base curve fluorescein patterns in keratoconus. Optom Vis Sci 2001;78:589-598.

7. Gordon MO, Schechtman KB, Davis LJ, McMahon TT, Schornack J, Zadnik K. Visual acuity repeatability in keratoconus: impact on sample size. Collaborative Longitudinal Evaluation of Keratoconus (CLEK) Study Group. Optom Vis Sci 1998;75:249-257.

8. Raasch TW, Schechtman KB, Davis LJ, Zadnik K. Repeatability of subjective refraction in myopic and keratoconic subjects: results of vector analysis. Ophthalmic Physiol Opt 2001;21:376-383.

9. Davis LJ, Schechtman KB, Begley CG, Shin JA, Zadnik K. Repeatability of refraction and corrected visual acuity in keratoconus. The CLEK Study Group. Collaborative Longitudinal Evaluation of Keratoconus. Optom Vis Sci 1998;75:887-896.

10. McMahon TT, Anderson RJ, Joslin CE, Rosas GA. Precision of three topography instruments in keratoconus subjects. Optom Vis Sci 2001;78:599-604.

11. McMahon TT, Anderson RJ, Roberts C, et al. Repeatability of corneal topography measurement in keratoconus with the TMS-1. Optom Vis Sci 2005;82:405-415.

12. Szczotka LB, Barr JT, Zadnik K. A summary of the findings from the Collaborative Longitudinal Evaluation of Keratoconus (CLEK) Study. CLEK Study Group. Optometry 2001;72:574-584.

13. Zadnik K, Barr JT, Edrington TB, et al. Baseline findings in the Collaborative Longitudinal Evaluation of Keratoconus (CLEK) Study. Invest Ophthalmol Vis Sci 1998;39:2537-2546.

14. Fink BA, Wagner H, Steger-May K, et al. Differences in keratoconus as a function of gender. Am J Ophthalmol 2005;140:459-468.

15. Zadnik K, Barr JT, Edrington TB, et al. Corneal scarring and vision in keratoconus: a baseline report from the Collaborative Longitudinal Evaluation of Keratoconus (CLEK) Study. Cornea 2000;19:804-812.

16. Barr JT, Schechtman KB, Fink BA, et al. Corneal scarring in the Collaborative Longitudinal Evaluation of Keratoconus (CLEK) Study: baseline prevalence and repeatability of detection. Cornea 1999;18:34-46.

17. Barr JT, Zadnik K, Wilson BS, et al. Factors associated with corneal scarring in the Collaborative Longitudinal Evaluation of Keratoconus (CLEK) Study. Cornea 2000;19:501-507.

18. Barr JT, Wilson BS, Gordon MO, et al. Estimation of the incidence and factors predictive of corneal scarring in the Collaborative Longitudinal Evaluation of Keratoconus (CLEK) Study. Cornea 2006;25:16-25.

19. Zadnik K, Barr JT, Gordon MO, Edrington TB. Biomicroscopic signs and disease severity in keratoconus. Collaborative Longitudinal Evaluation of Keratoconus (CLEK) Study Group. Cornea 1996;15:139146.

20. Zadnik K, Steger-May K, Fink BA, et al. Between-eye asymmetry in keratoconus. Cornea 2002;21:671-679.

21. Nichols JJ, Steger-May K, Edrington TB, Zadnik K. The relation between disease asymmetry and severity in keratoconus. Br J Ophthalmol 2004;88:788-791.
22. Shen JF, McMahon T'T, Cheng EL, et al. Lysosomal hydrolase staining of conjunctival impression cytology specimens in keratoconus. Cornea 2002;21:447-452

23. Maeda N, Fujikado T, Kuroda T, et al. Wavefront aberrations measured with Hartmann-Shack sensor in patients with keratoconus. Ophthalmology 2002;109:1996-2003.

24. Liu E, Slomovic AR. Indications for Penetrating Keratoplasty in Canada, 1986-1995. Cornea 1997;16:414-419.

25. Amaral Cde S, Duarte JY, Silva PL, et al. Indications for penetrating keratoplasty in Pernambuco. Arq Bras Oftalmol 2005;68:635-637.

26. Zhang $\mathrm{C}, \mathrm{Xu}$ J. Indications for penetrating keratoplasty in East China, 1994-2003. Graefe's Archive for Clinical and Experimental Ophthalmology 2005;243:1005-1009.

27. Kang PC, Klintworth GK, Kim T, et al. Trends in the Indications for Penetrating Keratoplasty, 1980-2001. Cornea 2005;24:801-803.

28. Weed $\mathrm{KH}, \mathrm{McGhee} \mathrm{CN}$. Referral patterns, treatment management and visual outcome in keratoconus. Eye 1998;12:663-668.

29. Tuft SJ, Moodaley LC, Gregory WM, Davison CR, Buckley RJ. Prognostic factors for the progression of keratoconus. Ophthalmology 1994;101:439-447.

30. Reeves SW, Stinnett S, Adelman RA, Afshari NA. Risk Factors for Progression to Penetrating Keratoplasty in Patients With Keratoconus. American Journal of Ophthalmology 2005;140:607-611.

31. Caporossi A, Balestrazzi A, Simi C, et al. Manual Deep Lamellar Keratoplasty: Alternative Methods and Air-Guided Technique. Transplantation Proceedings 2005;37:2697-2701.

32. Bilgihan K, Ozdek SC, Sari A, Hasanreisoglu B. Microkeratome-assisted lamellar keratoplasty for keratoconus: stromal sandwich. Journal of Cataract \& Refractive Surgery 2003;29:1267-1272.

33. Busin M, Zambianchi L, Arffa RC. Microkeratome-Assisted Lamellar Keratoplasty for the Surgical Treatment of Keratoconus. Ophthalmology 2005;112:987-997.

34. Dupuy FP, Salvoldelli M, Robert AM,et al. Chemotactic penetration of keratocytes in ePTFE polymer in vitro. J Biomed Mater Res 2001;56:487493.

35. Bleckmann H, Holak S. Preliminary results after implantation of four AlphaCor artificial corneas. Graefe's Archive for Clinical and Experimental Ophthalmology 2006;244:502-506.

36. Alio JL, Artola A, Ruiz-Moreno JM, et al. Changes in keratoconic corneas after intracorneal ring segment explantation and reimplantation. Ophthalmology 2004;111:747-751.

37. Alio JL, Artola A, Hassanein A, et al. One or 2 Intacs segments for the correction of keratoconus. Journal of Cataract \& Refractive Surgery 2005;31:943-953

38. Hellstedt T, Makela J,Uusitalo R, et al. Treating Keratoconus With Intacs Corneal Ring Segments. Journal of Refractive Surgery 2005;21:236-246.

39. Hladun L, Harris M. Contact lens fitting over intrastromal corneal rings in a keratoconic patient. Optometry 2004;75:48-54.

40. Boxer Wachler BS, Christie JP, Chandra NS, et al. Intacs for keratoconus. Ophthalmology 2003;110:1031-1040.

41. Colin J, Cochener B, Savary G,et al. INTACS inserts for treating keratoconus: One-year results. Ophthalmology 2001;108:1409-1414.

42. Kwitko S, Severo NS. Ferrara intracorneal ring segments for keratoconus. Journal of Cataract \& Refractive Surgery 2004;30:812-820.

43. Levinger S, Pokroy R. Keratoconus Managed With Intacs: One-Year Results. 2005:123:1308-1314.

44. Siganos D, Ferrara P, Chatzinikolas K, et al. Ferrara intrastromal corneal rings for the correction of keratoconus. Journal of Cataract \& Refractive Surgery 2002;28:1947-1951.

45. Tunc Z, Devici N, Sener B, Bahcecioglu H. [Corneal ring segments (INTACS) for the treatment of asymmetrical astigmatism of the keratoconus. Follow-up after 2 years]. J Fr Ophtalmol 2003;26:824-830

46. Mularoni A, Torreggiani A, di Biase A, et al. Conservative Treatment of Early and Moderate Pellucid Marginal Degeneration: A new refractive approach with intracorneal rings. Ophthalmology 2005;112:660-666.

47. Miranda D, Sartori M, Francesconi C, et al. Ferrara intrastromal corneal ring segments for severe keratoconus. J Refract Surg 2003;19:645-653. 
48. Kasparova EA. [Pathogenetic basis for treatment of primary keratoconus by a combined method of excimer laser surgery (combination of photorefraction and phototherapeutic keratectomy)]. Vestn Oftalmol 2002;118:21-25.

49. Kasparova EA, Kasparov AA. Six-year experience with excimer laser surgery for primary keratoconus in Russia. J Refract Surg 2003;19:S250254.

50. Tamayo Fernandez GE, Serrano MG. Early clinical experience using custom excimer laser ablations to treat irregular astigmatism. Journal of Cataract \& Refractive Surgery 2000;26:1442-1450.

51. Tamayo GE, Serrano MG. Treatment of irregular astigmatism and keratoconus with the VISX C-CAP method. Int Ophthalmol Clin 2003;43:103-110.

52. Colin J, Simonpoli S. [Keratoconus: current surgical options]. J Fr Ophtalmol 2005;28:205-217.

53. Rowsey JJ, Gills JP, Gills III P. Treating Keratoconus with Astigmatic Keratotomy and Intraocular Lenses: A New Approach. SO - International Ophthalmology Clinics Summer 2003;43(3):81-92.

54. Colin J, Velou S. Implantation of intacs and a refractive intraocular lens to correct keratoconus. Journal of Cataract \& Refractive Surgery 2003;29:832-834

55. Budo C, Bartels MC, van Rij G. Implantation of Artisan Toric Phakic Intraocular Lenses for the Correction of Astigmatism and Spherical Errors in Patients With Keratoconus. Journal of Refractive Surgery 2005;21:218-222

56. Wollensak G, Spoerl E, Seiler T. Riboflavin/ultraviolet-a-induced collagen crosslinking for the treatment of keratoconus. American Journal of Ophthalmology 2003;135:620-627.

57. Wollensak G, Spoerl E, Wilsch M, Seiler T. Endothelial cell damage after
riboflavin-ultraviolet-A treatment in the rabbit. Journal of Cataract \& Refractive Surgery 2003;29:1786-1790.

58. Wollensak G, Sporl E, Reber F,et al. Corneal endothelial cytotoxicity of riboflavin/UVA treatment in vitro. Ophthalmic Res 2003;35:324-328.

59. Wollensak G, Spoerl E, Wilsch M, Seiler T. Keratocyte Apoptosis After Corneal Collagen Cross-linking Using Riboflavin/UVA Treatment. Cornea 2004;23:43-49.

60. Kampmeier J, Behrens A, Wang Y, et al. Inhibition of Rabbit Keratocyte and Human Fetal Lens Epithelial Cell Proliferation by RetrovirusMediated Transfer of Antisense Cyclin G1 and Antisense MAT1 Constructs. Hum Gene Ther 2000:11:1-8.

61. Mohan RR, Schultz GS, Hong J-W, et al. Gene transfer into rabbit keratocytes using AAV and lipid-mediated plasmid DNA vectors with a lamellar flap for stromal access. Experimental Eye Research 2003;76:373383.

62. Oshima Y, Sakamoto T, Hisatomi T, et al. Targeted Gene Transfer to Corneal Stroma in vivo by Electric Pulses. Experimental Eye Research 2002;74:191-198.

63. Seitz B, Moreira L, Baktanian E, et al. Retroviral vector-mediated gene transfer into keratocytes in vitro and in vivo. American Journal of Ophthalmology 1998;126:630-639.

64. Wang D-A, Du H, Jaggar JH, et al. Injury-elicited differential transcriptional regulation of phospholipid growth factor receptors in the cornea. 2002:283:C1646-1654.

65. Dean DA, Byrd JN Jr, Dean BS. Nuclear targeting of plasmid DNA in human corneal cells. Current Eye Research 1999;19:66-75.

66. Yan X, Rong P. Influence on human keratocyte proliferation by cationic liposome mediated transfer of p21WAF1]. Zhonghua Yan Ke Za Zhi 2002;38:103-107.

\section{AEA OPTOMETRIC CRUISE SEMINARS 2008-2009}

COASTAL, 9/20/08-9/27/08, Sapphire Princess ${ }^{\circledR}$. Vancouver, Nanaimo, Victoria, San Francisco, Catalina Island, San Diego, Los Angeles.

From $\$ 799$

EASTERN CARIBBEAN, 1/25/09-2/1/09, Crown Princess ${ }^{\circledR}$. Ft. Lauderdale, Princess Cays, St. Maarten, St. Thomas, Grand Turk, Ft. Lauderdale. From $\$ 659$

HAWAII, 2/14-2/21/09, NCL Pride of America ${ }^{\circledR}$. Honolulu, Maui, Hilo, Kona, Nawiliwili, Honolulu. From \$1259 Valentine’s Day

CLASSIC SOUTHERN CARIBBEAN, 2/15/09-2/22/09,

Caribbean Princess $^{\circledR}$. San Juan, Barbados, St. Lucia, Antigua,

Tortola, St. Thomas, San Juan. From $\$ 909$.

Speakers: Janet Betchkal, MD \& Rick Bendel, MD

EASTERN CARIBBEAN, 3/14-3/21/09, Disney Magic ${ }^{\circledR}$

Port Canaveral, St. Maarten, St. Thomas, Castaway Cay, Port Canaveral.

From \$1169.00 Spring Break with Disney!

WESTBOUND TRANSATLANTIC CROSSING, 5/25-5/31/09,

Cunard Queen Mary $2^{\circledR}$. Southampton to New York.

Premium balconies from $\$ 1752$

WESTERN CARIBBEAN, 6/27/09-7/4/09, Disney Magic ${ }^{\circledR}$. Port Canaveral, Key West, Grand Cayman, Cozumel, Castaway Cay, Port Canaveral.

From \$1549 Canada Day

EASTERN CARIBBEAN/BERMUDA, 6/29/09-7/8/09, Caribbean Princess ${ }^{\circledR}$. New York City, Bermuda (West End), San Juan, St. Thomas, Grand Turk, New York City. From \$1329 Canada Day

GULF OF ALASKA, 6/29/09-7/6/09, Coral Princess ${ }^{\circledR}$. Vancouver, Ketchikan, Juneau, Skagway, Glacier Bay National Park, College Fjord, Anchorage. From \$1009 Canada Day
HAWAII, 7/4/09-7/11/09, NCL Pride of America ${ }^{\circledR}$. Honolulu, Maui, Hilo, Kona, Nawiliwili, Honolulu. From \$1409

CLASSIC GRAND MEDITERRANEAN, 7/15/09-

7/27/09 Ruby Princess ${ }^{\circledR}$. Barcelona, Monte Carlo, Florence/Pisa, Rome, Naples/Capri, Mykonos, Istanbul, Kusadasi, Athens, Venice. From \$2240 Speaker: Dr. Paul Ajamian

BLUE DANUBE DISCOVERY RIVER CRUISE, 7/20-7/27/09, Amadeus Waterways Amadante ${ }^{\circledR}$. Budapest, Bratislava, Vienna, Durnstein-Melk, Linz-Passau, Regensburg, Nuremberg-Carlsbad-Prague. Optional 2 night pre-cruise stay in Budapest and/or 3 night post-cruise stay in Prague. Cruise fare INCLUDES wines $\mathrm{w} /$ dinner and most shore excursions! From \$2299 cruise only. Speaker: Dr. Robert Wooldridge

MEDITERRANEAN, 7/27-8/309, MSC Splendida ${ }^{\circledR}$. Barcelona, Tunis, Malta, Messina, Civitavecchia (Rome), Genoa, Marseille, Barcelona. KIDS 17 AND UNDER SAIL FREE AS $3^{\text {rd }} \& 4^{\text {th }}$ IN A CABIN

From \$1299. Speaker: Dr. Harue Marsden

Early booking discounts or regional promotions may apply. Call for lowest current price. Visit cruise line websites for terms, conditions, and definitions which will apply to all bookings. Fares are cruise only, per person, USD, based on double occupancy, capacity controlled and subject to availability. Government fees and taxes, fuel supplement are additional.

(C) 2006 Princess Cruises. Ships Registry: Bermuda /NCL Pride of America ${ }^{\oplus}$ Registry: The United States of America

Disney Magic ${ }^{\oplus}$ Registry: The Bahamas/Cunard Queen Mary $2^{\oplus}$ Registry: Britain Amadeus Waterways Amadante ${ }^{\oplus}$ Registry: Switzerland/MSC Orchestra ${ }^{\circledast}$ Registry: Panama

AEA CRUISES: DR. MARK ROSANOVA, PRESIDENT

More than a travel agent, your colleague \& innovative partner in Cruise Seminars since 1995.

Sponsored by the Illinois Optometric Association and Advanced Eyecare Associates 10-12 hours of COPE approved lectures per seminar

Visit us at www.OptometricCruiseSeminars.com, email aeacruises@aol.com or call us at 1-888-6386009.

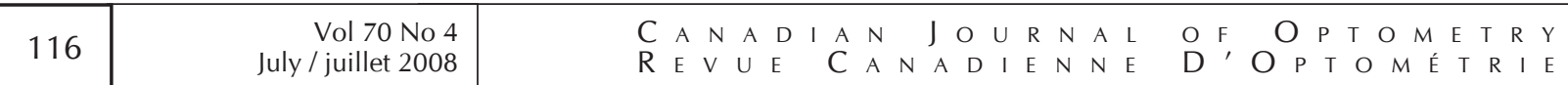

